\title{
A selective denoising method to remove speckle noise
}

\author{
Alex F. de Araujo \& João Manuel R. S. Tavares \\ Instituto de Engenharia Mecânica e Gestão Industrial, Faculdade de Engenharia, Universidade do Porto, $R$. \\ Dr Roberto Frias s/n, 4200-465 - Porto, PORTUGAL \\ fa.alex@gmail.com, tavares@fe.up.pt
}

\author{
Christos E. Constantinou \\ Department of Urology, School of Medicine Stanford University, Stanford, CA, USA \\ constantinou@stanfordalumni.org
}

\begin{abstract}
Speckle noise strongly affects the images acquired by ultrasound scans and reduces the efficiency of computational methods to extract and understand the features presented in the images. Trying to overcome this problem, a selective denoising method is proposed based on average filtering and on the radiation intensity associated to the input image pixels. Experimental tests were done using a set of simulated ultrasound images and a real ultrasound image sequence, and their statistical analysis confirmed that the proposed method has good ability to reduce speckle noise in ultrasound images.
\end{abstract}

\section{INTRODUCTION}

The presence of speckle noise in ultrasound images is common and strongly interferes in detecting and analyzing the anatomical structures in these images. The main type of noise found in ultrasound images appears due to the overlapping and deviating of the sound waves emitted and received by the ultrasound sensors. Hence, the study of techniques to reduce this type of noise is important as it allows the understanding of the corrupted images more efficiently.

Several computational image processing techniques have been proposed to remove image noise, including techniques based on Gaussian filters [1, $2]$, differential equations $[3,4,5,6]$ and multiresolution processing [7]. Usually, the speckle noise is more complex to be removed than Gaussian noise; for this reason, it is common to represent the speckle noise as additive noise. Thus, there are various works that try to remove speckle noise based on approaches originally developed to smooth images affected by additive noise.

Here, a novel selective smoothing method for images affected by speckle noise is proposed. The method uses the radiation intensity of the pixels of the image to be smoothed, and tries to find the pixels that belong to potential contours in order to perform a selective image smoothing using average filters with different intensities.

This paper is organized as follows: the proposed smoothing method is described in the next section, the experimental results and their discussion are pre- sented in section 3 , followed by the final considerations in the last section.

\section{PROPOSED METHOD}

The new denoising method is based on selective average filtering of the input image using average filters, and defines the intensity addressed by each filter according to the radiation accumulated by each image pixel from its neighbor pixels.

The method starts the noise removal process by weakly smooth the input image using an average filter with a $3 \times 3$ convolution window, which avoids the loss of important details presented. In the next step, the radiation intensity received by each image pixel is computed by summing all interferences caused by the neighbor pixels, using the following equation:

$$
R_{i}=\sum_{i=1}^{\frac{w}{2}}\left[\left(\frac{I_{i}}{4 \pi r^{2}}\right)-T_{m}\right],
$$

where $I_{i}$ is the pixel $i$ of image $I$ and $T_{m}$ is a regulating term. The radiation intensity is computed in a circular region of radius $r$, which starts with value equal 1 (one) and grows until $\frac{w}{2}$ pixels, where $w$ is the width of the input image. In this computation, only are considered the radiations associated to the neighbor pixels at the same line, column and diago- 
nals of $I_{i}$. As such, the influence of other neighbor pixels is discarded since it does not affect considerably $I_{i}$ in the main directions and so the computation cost can be reduced.

After calculating the radiation affecting each pixel of the original image, the method tries to estimate the pixels with high probability to be noise in order to perform a stronger smoothing at those pixels. From our study, we have concluded that pixels receiving positive radiations have higher probability to be affected by noise or to belong to homogeneous regions. So, a stronger smoothing is performed at those pixels using an average filter with a $25 \times 25$ convolution window.

\section{EXPERIMENTAL RESULTS AND DISCUSSION}

To verify the efficiency of the method proposed, tests were performed using a set of synthetic images built using the computational simulator Field II [8, 9] and a set of real images acquired using a commercial medical ultrasound scan.

Initial tests were performed using a set of synthetic images, and the smoothed images were compared against the original ones by computing the MSSIM (Mean Structure Similarity Index Map) and ENL (Effective Number of Looks) indexes. It should be noted that the smoothing results are superior as the MSSIM and the ENL indexes are higher. In Figure 1 , the results of smoothing one synthetic image that was submitted to ultrasound imaging simulation using Field II are presented. Comparing the resultant images and the index values, it can be realized the efficiency of the proposed method.

In a second test, some real ultrasound images were processed by the same smooth methods used in the first test, and the ENL index was adopted to measure the quality of the resultant images. As indicated in Figure 2, the ENL index value is higher for the proposed method, which means that the method achieved a superior smoothing.

The depicted regions in the images presented in Figures 1 and 2 (surrounded by the white rectangles), allow to confirm visually that the proposed method was able to perform a strong smoothing in the homogeneous regions, making these regions more similar than the regions with relevant structure borders. Hence, the results confirm that the denoising method proposed removes successfully speckle noise in ultrasound images, being restored the homogeneous regions and preserved the contours presented.

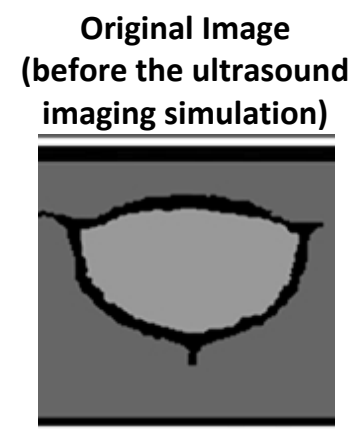

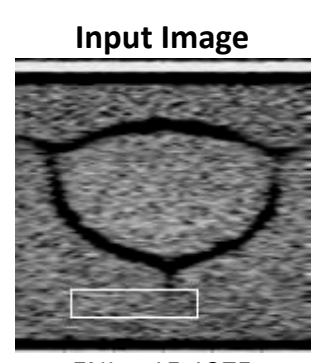

$\mathrm{ENL}=15.1275$

MSSIM $=0.3021$

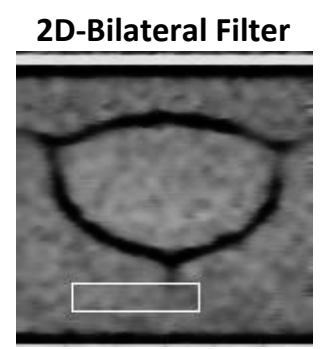

$\mathrm{ENL}=66.1123$

MSSIM $=0.6112$

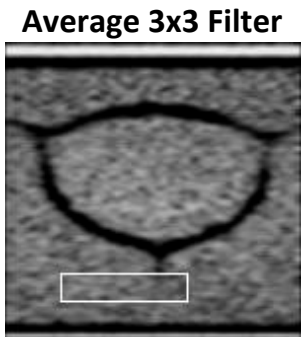

$E N L=35.4988$

MSSIM $=0.4404$

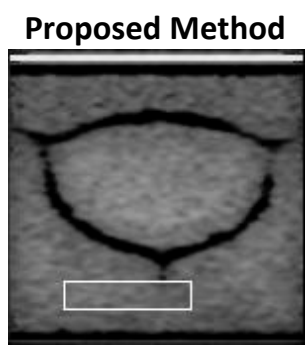

$E N L=87.1674$

MSSIM $=0.6250$
Figure 1: Results obtained using the average filter with a 3x3 convolution window, 2D-Bilateral Filter, and the denoising method proposed, applied on a synthetic image. The image quality indexes indicated in bold identify the method that achieves the best result.

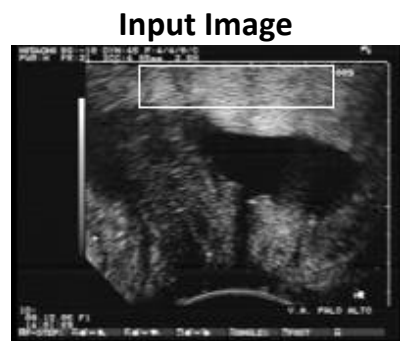

$E N L=16.6086$

2D-Bilateral Filter

$E N L=24.8887$

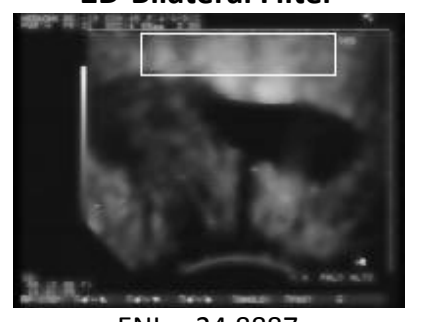

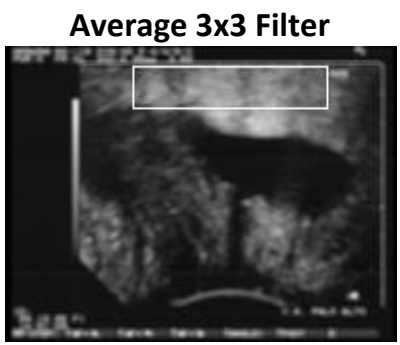

$\mathrm{ENL}=19.5774$

Proposed Method

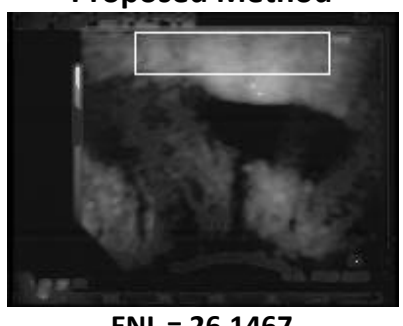

$E N L=26.1467$
Figure 2: Results obtained using the average filter with a $3 x 3$ convolution window, 2D-Bilateral Filter, and the denoising method proposed, applied on an ultrasound image. The image quality indexes indicated in bold identify the method that achieves the best result. 
In Figure 3, five images randomly selected from a real ultrasound image sequence and the correspondent images smoothed by the proposed method are shown.
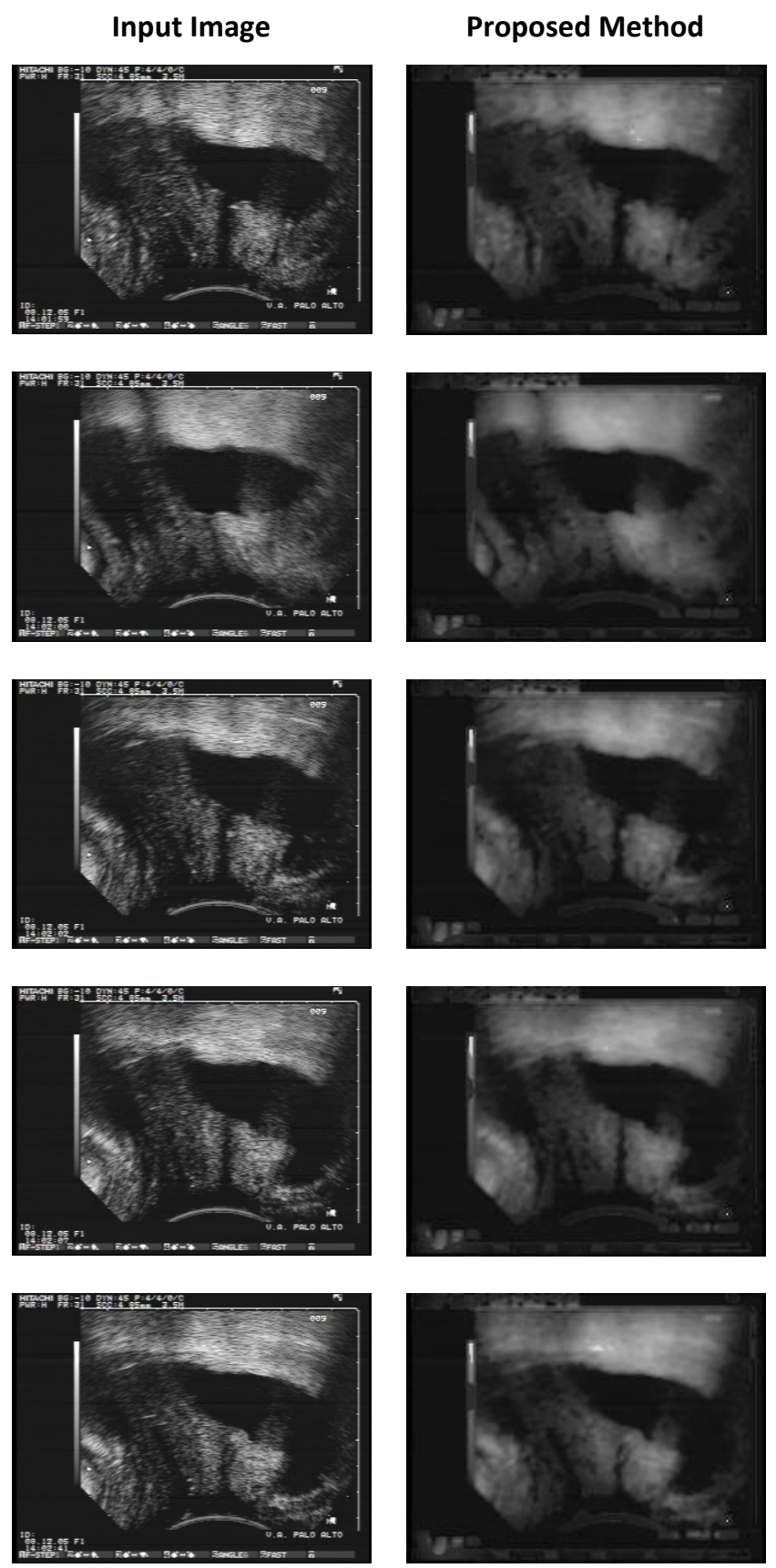

Figure 3: Results obtained using the proposed denoising method applied in real ultrasound images.

\section{CONCLUSIONS}

A novel method to smooth images affected by speckle noise, which is based on average filters to perform selective smoothing, was proposed. Experimental tests were accomplished using simulated and real ultrasound images in order to study the efficiency of the denoising method proposed in reducing the interferences caused by speckle noise on this kind of images. The results demonstrated that the method proposed is promising and efficient, as was confirmed by the image quality indexes computed.

\section{Acknowledgments}

The first author would like to thank his $\mathrm{PhD}$ grant from Fundação para a Ciência e a Tecnologia (FCT) in Portugal with reference SFRH/BD/61983/2009.

This work was partially done in the scope of the project "Methodologies to Analyze Organs from Complex Medical Images - Applications to Female Pelvic Cavity", with reference PTDC/EEACRO/103320/2008, financially supported by FCT.

\section{References}

[1] H.-Y. Yang, X.-Y. Wang, T.-X. Qu, and Z.$\mathrm{K}$. Fu, "Image denoising using bilateral filter and gaussian scale mixtures in shiftable complex directional pyramid domain," Computer and Electrical Engineering, vol. 37, no. 5, pp. 656-668, 2011.

[2] A. Adams, N. Gelfand, J. Dolson, and M. Levoy, "Gaussian kd-trees for fast highdimensional filtering," ACM Transacyions on Graphics, vol. 28, no. 3, pp. 21:1-21:12, 2009.

[3] C. A. Z. Barcelos, M. Boaventura, and E. C.S. Jr., "A well-balanced flow equation for noise removal and edge detection." IEEE Transactions on Image Processing, vol. 12, no. 7, pp. 751763, 2003.

[4] G. Aubert and J.-F. Aujol, "A variational approach to removing multiplicative noise." SIAM Journal of Applied Mathematics, vol. 68, no. 4, pp. 925-946, 2008.

[5] Y.-M. Huang, M. K. Ng, and Y.-W. Wen, “A new total variation method for multiplicative noise removal," SIAM Journal on Imaging Sciences, vol. 2, no. 1, pp. 20-40, 2009.

[6] O. Ghita and P. F. Whelan, "A new gvf-based image enhancement formulation for use in the presence of mixed noise," Pattern Recognition, vol. 43, no. 8, pp. 2646-2658, 2010.

[7] M. Jansen, Noise Reduction by Wavelet Thresholding, ser. Lecture Notes in Statistics. Springer, 2001.

[8] J. Jensen, "Field: A program for simulating ultrasound systems," in Proceedings of the 10th Nordic-Baltic Conference on Biomedical Imaging, vol. 34. Medical \& Biological Engineering \& Computing, 1996, pp. 351-353.

[9] J. Jensen and N. B. Svendsen, "Calculation of pressure fields from arbitrarily shaped, apodized, and excited ultrasound transducers," IEEE Transactions on Ultrasonics, Ferroelectrics and Frequency Control, vol. 39, no. 1, pp. 262-267, 1992. 
